\title{
Integration of collagen into chitosan blend film composites: physicochemical property aspects for pharmaceutical materials
}

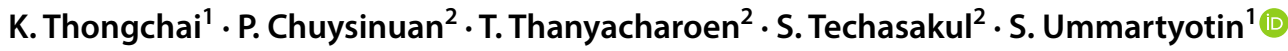

Received: 12 October 2019 / Accepted: 15 January 2020 / Published online: 22 January 2020

(c) Springer Nature Switzerland AG 2020

\begin{abstract}
Chitosan and collagen-based hydrogel composites were successfully prepared at chitosan:collagen volume ratio of 1:1, 1:3, 1:5, and 1:10. The structural, thermal, morphological, swelling, mechanical characteristics were investigated, and in vitro degradation testing was conducted. Tetraethyl orthosilicate (TEOS) was used as a crosslinking agent between the chitosan and collagen chains. The $\mathrm{NH}$ group of both chitosan and collagen can form crosslinks with the $\mathrm{OH}$ groups of TEOS. XRD reported that no significant peak of crystallinity was observed during the integration of collagen into the chitosan film. The surface of the composite was rougher than that of the collagen. The composites were thermally stable up to $200^{\circ} \mathrm{C}$. The glass transition temperature slightly decreased in the presence of collagen. The tensile strength significantly decreased, while the elongation at break significantly increased, when collagen was integrated into the chitosan film. The collagen conferred strong swelling and rapid degradation on the composite. The chitosan/collagen composite films developed in this study have potential applications as pharmaceutical materials.
\end{abstract}

\section{Graphic abstract}
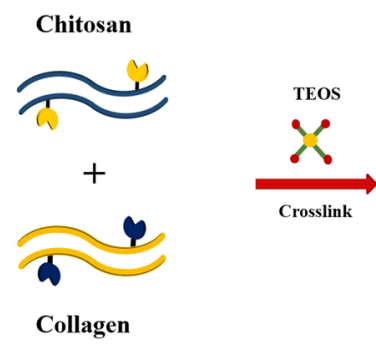

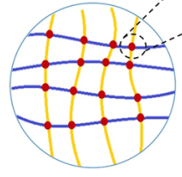

Hydrogel

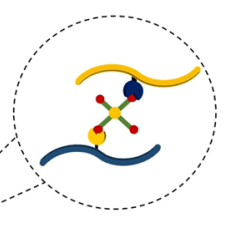

Keywords Chitosan $\cdot$ Collagen $\cdot$ Hydrogel $\cdot$ Composite

\section{Introduction}

In recent years, the development of safe and biocompatible biomaterials has been a key goal of medical technology research. Numerous types of biomaterials have been investigated as replacements for synthetic material. The practicality of biomaterials such as cellulose and its derivatives, chitin-chitosan, polycaprolactone, and polylactic acid as active materials is being extensively investigated. Biomaterials have been used in applications including

S. Ummartyotin, sarute@tu.ac.th | ${ }^{1}$ Department of Materials and Textile Technology, Faculty of Science and Technology, Thammasat University, Patumtani, Thailand. '2 Laboratory of Organic Synthesis, Chulabhorn Research Institute, Bangkok, Thailand. 
drug delivery, cosmetics, regenerative medicine, and as food additives. Given the support for environmental policies, academic research and industrial commercialization have encouraged development of biomaterials and bioprocesses.

Chitosan is a widely investigated biomaterial [1]. From the fundamental perspective, chitosan is a linear polysaccharide composed of randomly distributed $\beta$ - $(1 \rightarrow 4)$-linked D-glucosamine and N-acetyl-D-glucosamine. This biomaterial can be prepared by treating the chitin shells of other crustaceans. The utilization of chitosan and its modification have been active research topics in many areas including food additives, antimicrobial agents, wound dressing, and drug delivery systems [2,3]. The development of chitosan and synthetic polymers for hydrogel composites has been investigated. Onat et al. [4] and Han et al. [5] developed the multifunctional layer-bylayer characteristics of three composites: chitosan, polyethylene glycol, and polyethylene oxide-based hydrogel. Thanyacharoen et al. [6] developed chitosan and polyvinyl alcohol-based hydrogel composites. The hydrogel provides the useful characteristic of controlled release for gallic acid. Furthermore, chitosan and polyvinyl alcoholbased hydrogel composites have been used as the matrix phase for the loading of bioactive molecules prepared from Oryza sativa L. [7]. The development of chitosan and bio-based polymers prepared from naturally-occurring resources are designed to replace synthetic polymer. Nguyen et al. [8] and George et al. [9] prepared hydrogel with gelatin and cellulose, respectively. With the expectation that chitosan hydrogel would be efficient, crosslinking between chitosan and polymer has been investigated [10, 11 ] to enhance flexibility and thermal resistance.

However, chitosan and collagen hydrogels have been investigated in the search for more efficient chitosanbased hydrogel for use in medical and pharmaceutical technology. Collagen is the main structural protein in the extracellular space of some connective tissue [12]. It supports cell attachment in vertebrates, interacts with cells, and regulates cell migration, proliferation, and survival [13]. Thus, inserting collagen into a chitosan hydrogel can enhance biocompatibility. A further challenge for collagen-based hydrogel is to make it suitable for physical crosslinking, for example by UV and $\gamma$ radiation. This can produce non-toxic hydrogel. Wang et al. [14] suggested that toxicity is a concern for human use, even though formaldehyde, glutaraldehyde, and genipin play essential roles in the chemical crosslinking process.

In the present work, we investigated the role of collagen in the preparation of chitosan-based hydrogel composite. Collagen was incorporated into a chitosan hydrogel and the physicochemical properties of the collagen/chitosan composites obtained were determined via Fourier transform infrared (FTIR) spectroscopy, differential scanning calorimetry (DSC), and x-ray diffraction (XRD). Scanning electron microscopy (SEM) was used to observe the morphology. Swelling behavior and degradation were studied.

\section{Experimental}

\subsection{Materials}

Chitosan (medium molecular weight, $75-85 \%$ degree of deacetylation) was purchased from Sigma Aldrich Co., Ltd. Collagen (molecular weight $\geq 80 \mathrm{~g} / \mathrm{mol}$ more than $90 \%$ protein) was purchased from Chemipan Corporation Co., Ltd. The $\mathrm{pH}$ was in the range 4-7. These materials were used as starting reagents. Tetraethyl orthosilicate (TEOS) was purchased from Sigma Aldrich Co., Ltd. and was utilized as a crosslinking agent. Acetic acid was purchased from Labscan Asia Co., Ltd. and was used as the solvent. All chemical reagents were used as received, without further purification.

\subsection{Methods}

\subsubsection{Preparation of chitosan and collagen hydrogel composite}

Chitosan and collagen-based hydrogel composite films were prepared using wet chemistry. Pristine chitosan film was prepared by casting chitosan solution on a petri dish. Chitosan and collagen films were similarly prepared by casting a chitosan and collagen solution, prepared by dissolving $1 \mathrm{~g}$ of chitosan powder in acetic acid (1\% v/v) and $1 \mathrm{~g}$ of collagen powder in deionized water. Setting was for $1 \mathrm{~h}$ at $50^{\circ} \mathrm{C}$ to ensure solubility. To prepare the soft composite, we mixed the chitosan and collagen solution, at volume ratios of $1: 1,1: 3,1: 5$, and $1: 10$. Only $2 \% \mathrm{vol} / \mathrm{vol}$ of TEOS was added to the mixture, which was then stirred at ambient temperature for $1 \mathrm{~h}$ to obtain a homogeneous solution. To prepare the composite film sheet, the solution was cast on a petri dish and dried at ambient temperature for a week. The composite film was stored in desiccator to prevent moisture absorption.

\subsubsection{Swelling test}

A gravimetric technique was used to determine the swelling behavior of the composite. The hydrogel was cut into $1 \mathrm{~cm} \times 1 \mathrm{~cm}$ squares and the mass was recorded as $W_{\text {dry }}$. The samples were then immersed in deionized water. After an appropriate time interval the specimens were removed from the solution, and excess water was removed using 
paper. The size of the specimens was measured. Five samples were used and statistical averages and standard deviations were derived. The swelling ratio was calculated using (Eq. 1).

$\mathrm{Q}(\mathrm{g} / \mathrm{g})=\left(\mathrm{W}_{\text {wet }}-\mathrm{W}_{\text {dry }}\right) / \mathrm{W}_{\text {dry }}$

where $W_{\text {wet }}$ is the weight of swollen hydrogel at the end of submersion and $W_{\text {dry }}$ is the initial weight of the dry hydrogel.

\subsubsection{Degradation test}

Composite squares of $1 \mathrm{~cm} \times 1 \mathrm{~cm}$ were immersed in $10 \mathrm{~mL}$ of deionized water at time intervals of $3,6,9,12$ and 15 days. Sample were removed and dried at $60^{\circ} \mathrm{C}$ at each time point. The percentage degradation was calculated using (Eq. 2).

Degradation $(\%)=\frac{\mathrm{Wt}}{\mathrm{W} 0} \times 100$,

where $W_{0}$ and $W_{t}$ are the weights of the sample before and after degradation, respectively.

\subsection{Instruments}

Surface morphology was determined using a scanning electron microscope (JEOL JSM-6400). The samples were placed on a stub coated with a thin layer of gold using a
JEOL JFC-1100E sputtering device. The crystal structure of the sample was investigated via XRD (Bruker D8 DISCOVER System) using nickel-filtered CuKa radiation. Diffraction patterns were recorded from $1^{\circ}$ to $50^{\circ}$. The chemical structure was determined using an FTIR spectrophotometer (SPECTRUM ONE, Perkin Elmer). The hydrogel composites were stored in a desiccator before analysis. Samples of $10 \mathrm{~mm} \times 10 \mathrm{~mm}$ were scanned from 400 to $4000 \mathrm{~cm}^{-1}$ at room temperature in an attenuated total reflectance mode at $4 \mathrm{~cm}^{-1}$ resolution. Thermal stability was evaluated via thermogravimetric analysis (TGA) (209 F3 Tarsus, NETZSCH). The samples were heated from room temperature to $600^{\circ} \mathrm{C}$ at $10^{\circ} \mathrm{C} / \mathrm{min}$ under a nitrogen atmosphere. DSC was used to evaluate the thermal properties, at a temperature range and heating rate of $0-250^{\circ} \mathrm{C}$ and $10^{\circ} \mathrm{C} /$ $\min$.

\section{Results and discussion}

\subsection{Physicochemical properties of chitosan and collagen-based hydrogel composites}

Chitosan was successfully integrated into collagen film via chemical crosslinking. TEOS was applied as a crosslinking agent for hydrogel formation. Figure 1 presents the FTIR spectra of the collagen and chitosan-based composites. The pristine chitosan was also analyzed as a control. The wavenumber at $3300-3700 \mathrm{~cm}^{-1}$ was attributed to
Fig. 1 FTIR spectra of chitosan and collagenbased hydrogel composites (a) pristine chitosan, (b) chitosan:collagen $=1: 1$, (c) chitosan:collagen $=1: 3,(d)$ chitosan:collagen $=1: 5$ and $(\mathrm{e})$ chitosan:collagen $=1: 10$

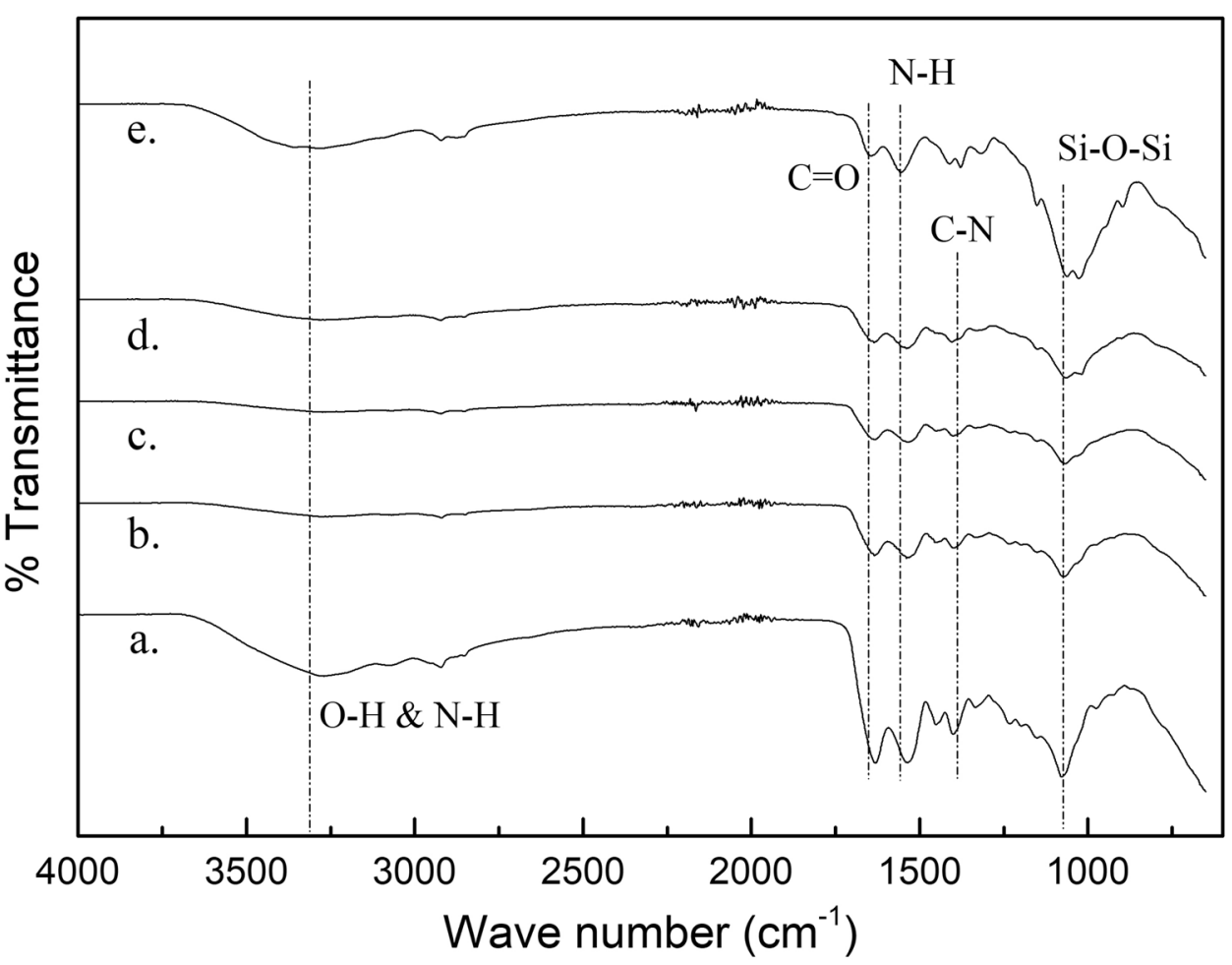

SN Applied Sciences 
$\mathrm{OH}$ - and $\mathrm{NH}$-stretching. This broad region is implicated in water adsorption onto the surface of collagen film (Kudo et al.) [15]. The spectra of all samples presented characteristic peaks at 1640 and $1540 \mathrm{~cm}^{-1}$. These were attributed to $\mathrm{C}=\mathrm{O}$ and $\mathrm{N}-\mathrm{H}$ stretching and attributed to protein amide. A characteristic peak at $1350 \mathrm{~cm}^{-1}$ was attributed to $\mathrm{C}-\mathrm{N}$ stretching, suggesting an imide bond (Beppu et al.) [16], and that at $1100 \mathrm{~cm}^{-1}$ to $\mathrm{Si}-\mathrm{O}-\mathrm{Si}$, probably due to crosslinking by TEOS. The formation of the chitosan and collagen-based hydrogel composite with TEOS as a crosslinking agent was due to hydrogen bonding between the amino groups on the chitosan and silanol groups on the TEOS. This was supported by the broad peak of $\mathrm{NH}$ stretching. The reaction of the collagen and crosslinking agent may account for the covalent bonding.

XRD was use to investigate the crystallinity of composites. Pristine chitosan and collagen were used as controls. Figure 2 shows the XRD patterns. The crystal structure of the chitosan was unaltered by the collagen. All samples presented an amorphous structure, with similar peak position and intensity. The collagen ratio did not affect the crystalline structure, probably because of water adsorption from the collagen chain.

The microstructural properties of the composites were investigated using scanning electron microscopy. Figure 3 shows the microstructure of the composites. Pristine chitosan film was used as control. The hydrogel presented a uniform network, independent of the ratio of collagen [17]. For the pristine chitosan, the surface was smooth. However, in case of composite, the rough surface was observed due to the presence of collagen. Phase

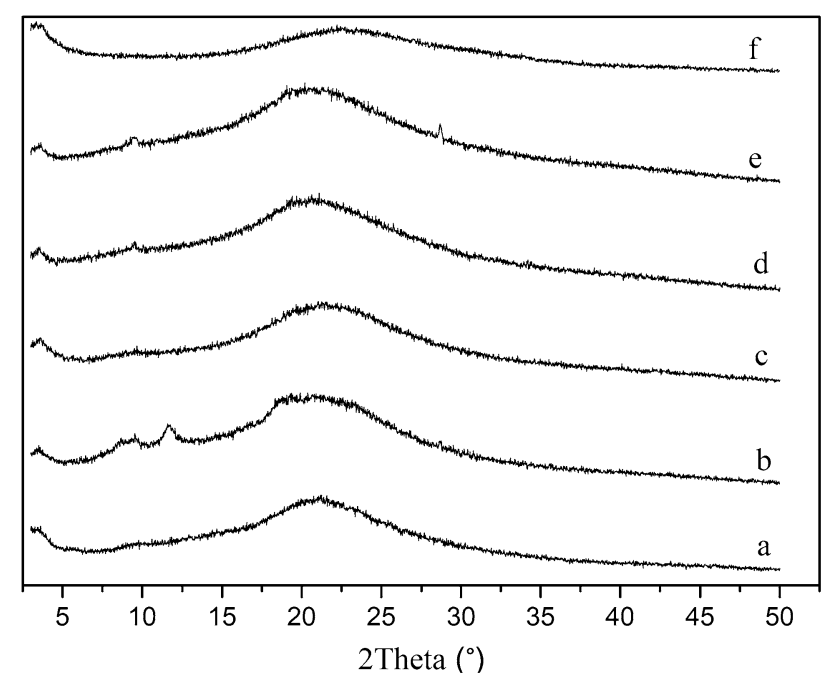

Fig. 2 XRD pattern of the chitosan and collagen-based hydrogel composites (a) pristine chitosan, (b) chitosan:collagen =1:1, (c) chitosan:collagen=1:3, (d) chitosan:collagen=1:5, (e) chitosan:collagen $=1: 10$ and $(f)$ pristine collagen separation occurred during the crosslinking of chitosan and collagen. This supported the findings of Gilarska et al. [18].

The thermal degradation of the composites was investigated using thermal gravimetric analysis, as shown in Fig. 4. Thermal degradation could be classified into three regions. The increase from room temperature to $200{ }^{\circ} \mathrm{C}$ accounted for only $10 \mathrm{wt} \%$ of weight loss. This was attributed to water evaporation from the hydrogel. This was followed by a broad region of weight loss between 200 and $400{ }^{\circ} \mathrm{C}$. This region involved pyrolysis. The hydrogel structure was organic and $\mathrm{CO}_{2}$ and $\mathrm{CO}$ evaporation took place in this stage. Thermal degradation became stable at temperatures exceeding $400^{\circ} \mathrm{C}$, and no was pyrolysis was observed. The composite was reduced to char and residue. However, weight loss increased in the presence of collagen. This was attributed to the preferential adsorption of water by the collagen. This thermal degradation mechanism was similar to that reported by Lewandowska et al. [19].

The thermal properties were also investigates using DSC. Figure 5 shows the DSC curve of the chitosan and collagen-based hydrogel composites. The glass transition temperatures were $107^{\circ} \mathrm{C}$ for pristine chitosan, $64^{\circ} \mathrm{C}$ for chitosan:collagen $1: 1,63^{\circ} \mathrm{C}$ for chitosan:collagen $1: 3,62^{\circ} \mathrm{C}$ for chitosan:collagen $1: 5,61{ }^{\circ} \mathrm{C}$ for chitosan:collagen $1: 10$. The glass transition temperature was slightly lower in the presence of collagen, perhaps reflecting the conformation of the chitosan and collagen chain [20]. However, the composite enthalpy was significantly decreased, attributed to the presence of TEOS. The crosslinking reaction between chitosan and collagen provided strong bonding along the chitosan and collagen chain.

The mechanical properties of the chitosan and collagen-based hydrogel composites were measured. Table 1 shows the tensile strength and elongation at the composite break. After collagen was integrated into the chitosan film, the tensile strength significantly decreased, whereas the elongation at break increased. This was due to the presence of collagen, which readily adsorbs water. A flexible composite was obtained, with hydrophilic.

\subsection{Preliminary investigation as pharmaceutical materials}

Swelling behavior is an important factor in the selection of pharmaceutical materials. Figure 6 shows the swelling of chitosan and collagen-based hydrogel composites, with pristine chitosan as control. The experiment was run for $6 \mathrm{~h}$. After $6 \mathrm{~h}$ of immersion in water, the hydrogel became dissolved. The weight of the swollen gel could not be determined. In structural terms, chitosan and collagen contain high amounts of the hydroxyl and amine groups, 


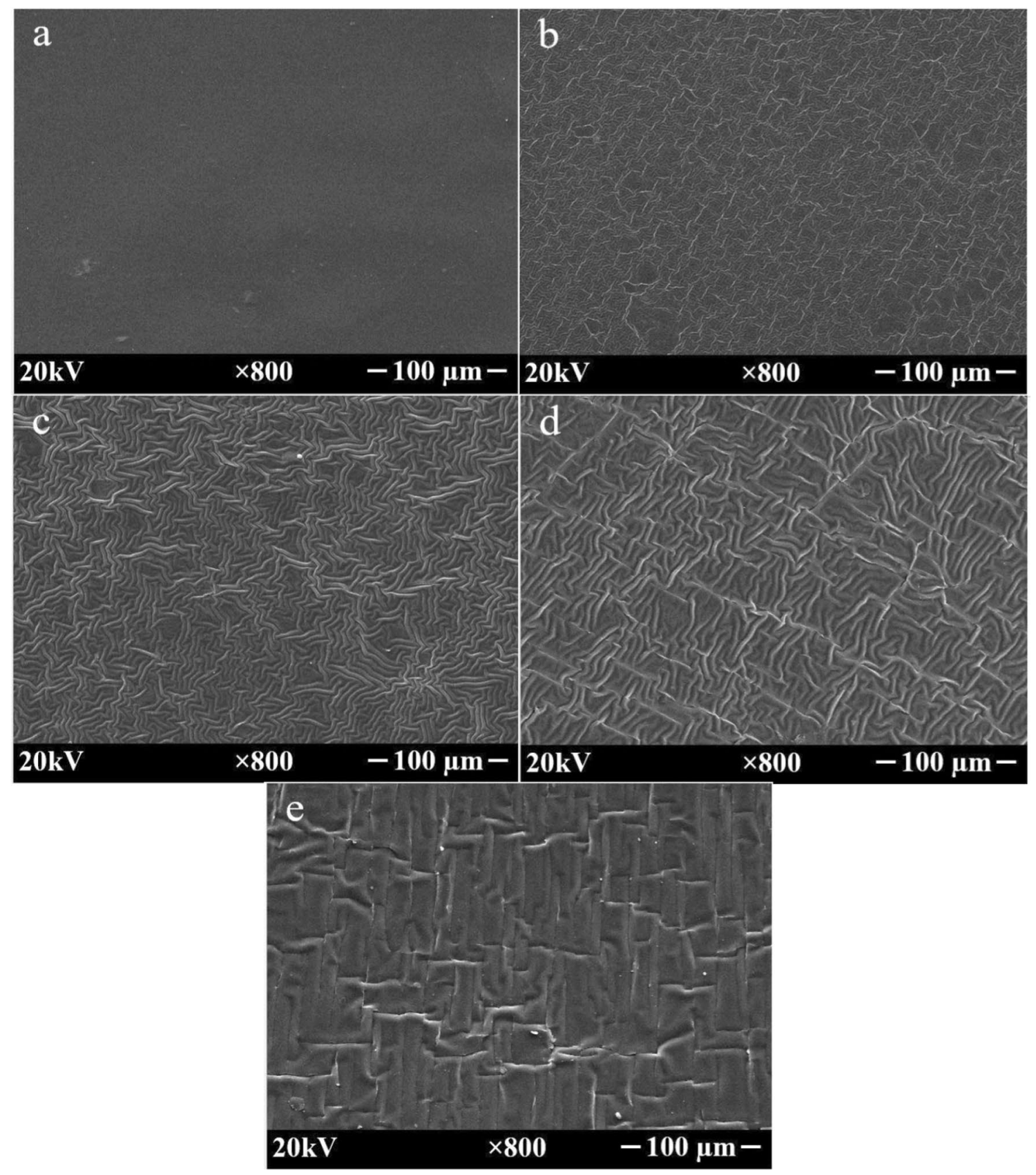

Fig. 3 Morphological properties of chitosan and collagen-based hydrogel composites (a) pristine chitosan, (b) chitosan:collagen=1:1, (c) chitosan:collagen $=1: 3$, (d) chitosan:collagen $=1: 5$ and $(e)$ chitosan:collagen $=1: 10$

making them strong hydrophilic [21]. Swelling was considerably enhanced when collagen was added to the chitosan film. This result strongly supported those reported by Kaczmarek et al. [22]. The pristine collagen exhibited a high percentage swelling. A high rate of swelling was observed over the first $30 \mathrm{~min}$, then becoming stable up to $6 \mathrm{~h}$.

A degradation test was conducted to determine the suitability of the chitosan and collagen-based hydrogel composites as medical materials. The degradation test was designed to quantify the lifetime. Previous studies have reported the in vitro degradation of chitosan and collagen-based hydrogel $[23,24]$. Figure 7 shows our in vitro degradation test of the chitosan and collagenbased hydrogel composites. The experiment was run for 15 days. Degradation over the first 3 days was significantly lower in the presence of collagen. Degradation was then stable from 3 day to 15 day. This was attributed to swelling, as shown in Fig. 6 . The presence of collagen 


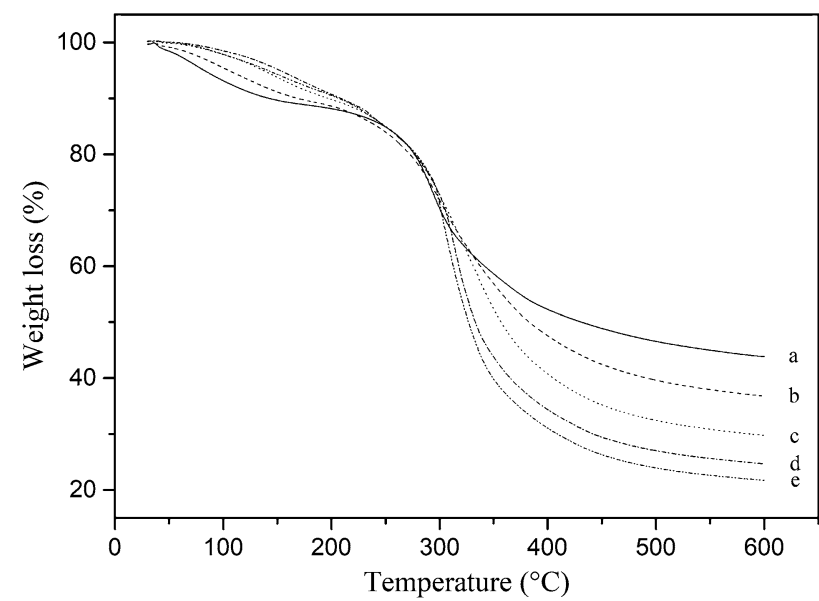

Fig. 4 Thermal decomposition behavior of chitosan and collagen-based hydrogel composites (a) pristine chitosan, $\begin{array}{lll}\text { (b) chitosan:collagen=1:1, } & \text { (c) chitosan:collagen=1:3, } & \text { (d) }\end{array}$ chitosan:collagen $=1: 5$ and $(\mathrm{e})$ chitosan:collagen $=1: 10$

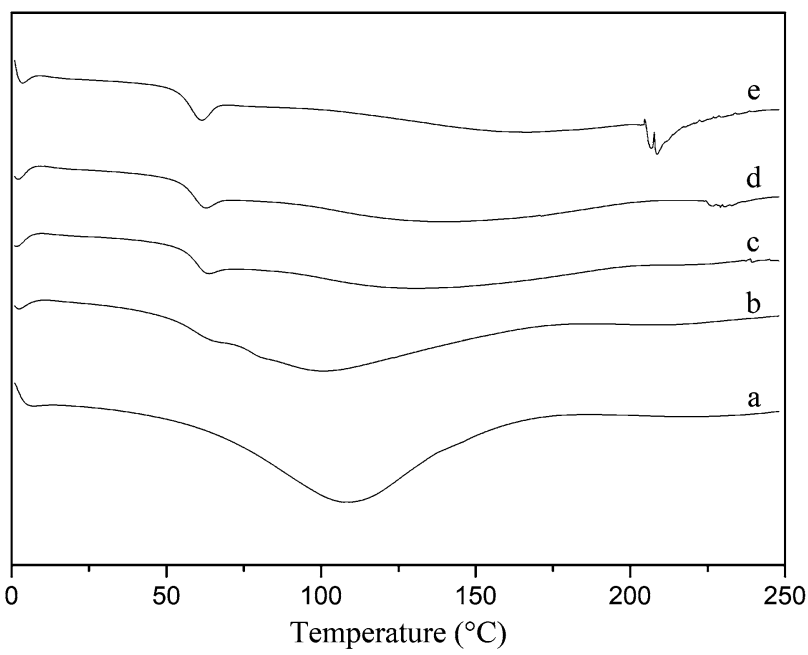

Fig. 5 DSC thermogram of chitosan and collagen-based hydrogel composites (a) pristine chitosan, (b) chitosan:collagen $=1: 1$, (c) chitosan:collagen=1:3, (d) chitosan:collagen=1:5 and (e) chitosan:collagen $=1: 10$

Table 1 Mechanical properties of chitosan and collagen-based hydrogel composites (CS/Coll)

\begin{tabular}{lcc}
\hline Film & Tensile strength $(\mathrm{MPa})$ & Elongation $(\%)$ \\
\hline CS & $66.00 \pm 8.61$ & $3.18 \pm 0.63$ \\
CS/Coll 1:1 & $52.49 \pm 7.92$ & $3.63 \pm 1.42$ \\
CS/Coll 1:3 & $28.21 \pm 5.38$ & $13.67 \pm 12.10$ \\
CS/Coll 1:5 & $10.34 \pm 1.97$ & $21.95 \pm 21.67$ \\
CS/Coll 1:10 & $6.35 \pm 1.97$ & $27.15 \pm 13.87$ \\
\hline
\end{tabular}

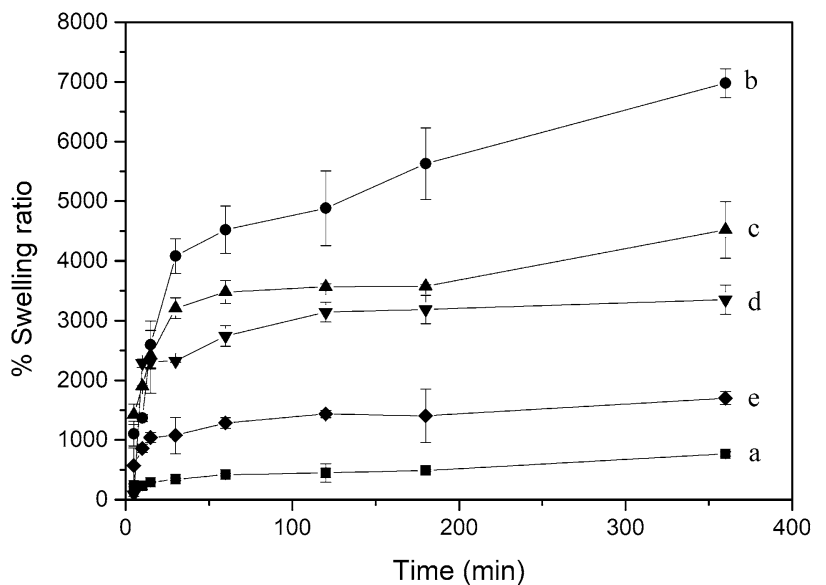

Fig. 6 Swelling behavior of chitosan and collagen-based hydrogel composites (a) pristine chitosan, (b) chitosan:collagen =1:1, (c) chitosan:collagen=1:3, (d) chitosan:collagen=1:5 and (e) chitosan: collagen $=1: 10$

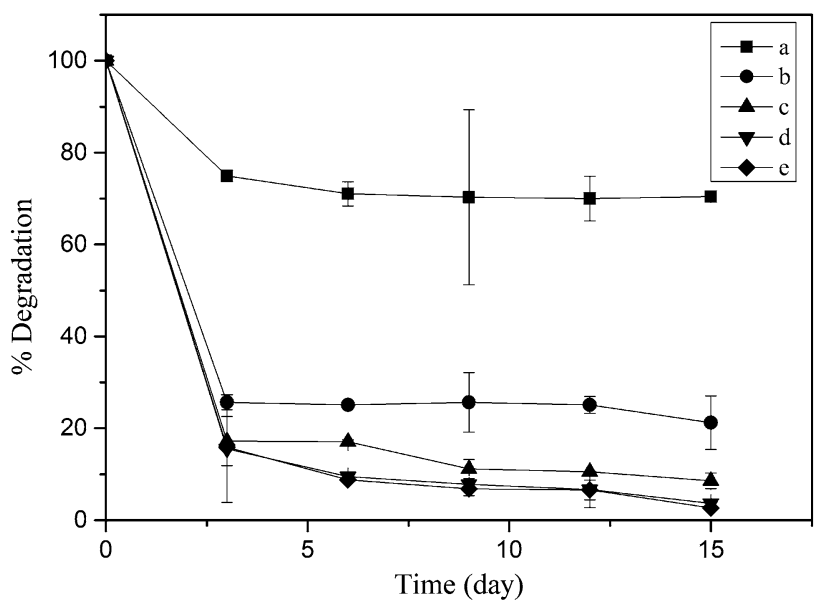

Fig. 7 Degradation behavior of chitosan and collagenbased hydrogel composites (a) pristine chitosan, (b) chitosan:collagen $=1: 1, \quad$ (c) chitosan:collagen $=1: 3$, chitosan:collagen $=1: 5$ and (e) chitosan:collagen $=1: 10$

can increase water adsorption, but the collagen itself becomes degraded when immersed in water. A similar result was reported by Liu et al. [25]. The presence of collagen would therefore affect the degradation of chitosan. Degradation was higher than in the pristine chitosan, with only a residual $20 \mathrm{wt} \%$ observed. Carbonyl polymers have high hydrolysis rates. As hydrolysis results in chain cleavage of polymers, their physical properties be significantly affected once hydrolysis begins. Hydrolysis can be tracked from the molecular weight of the material during its lifetime [26]. 


\section{Conclusions}

In this work, we studied the physicochemical properties of chitosan and collagen-based hydrogel composites, using TEOS as a crosslinking agent. The structural, thermal, morphological, and surface properties of the composites were characterized using FTIR, XRD, SEM, TGA, and DSC. The crosslinking reaction between collagen and chitosan molecule was occurred. With the presence of collagen, the morphological properties of composite became rough. Percent of residual and glass transition temperature were reduced to $20-40 \%$ and $60^{\circ} \mathrm{C}$ for composite. The presence of collagen in hydrogel composite can be provided the superiority on swelling behavior. Also, $20 \%$ of degradation was observed within 3 days. However, the addition of collagen decreased the tensile strength, and the elongation at break was slightly increased. The addition of collagen to chitosan-based hydrogel composite exhibited the excellent properties as pharmaceutical materials.

Acknowledgements The authors acknowledge the financial support provided by Thammasat University (Grant No. 2020). We are grateful for the space and research facilities provided by the Chulabhorn Research Institute. K. Thongchai sincerely appreciates the financial support from the National Research Council of Thailand for her study.

\section{Compliance with ethical standards}

Conflict of interest No conflict of interest for this article entitled "integration of collagen into chitosan blend film composites: physicochemical property aspects for pharmaceutical materials".

\section{References}

1. LogithKumar R et al (2016) A review of chitosan and its derivatives in bone tissue engineering. Carbohydr Polym 151:172-188

2. Pellá MCG et al (2018) Chitosan-based hydrogels: from preparation to biomedical applications. Carbohydr Polym 196:233-245

3. Hamedi $\mathrm{H}$ et al (2018) Chitosan based hydrogels and their applications for drug delivery in wound dressings: a review. Carbohydr Polym 199:445-460

4. Onat B et al (2019) Multifunctional layer-by-layer modified chitosan/poly(ethylene glycol) hydrogels. Eur Polym J 112:73-86

5. Han J et al (2011) Electrospinning of methoxy poly(ethylene glycol)-grafted chitosan and poly(ethylene oxide) blend aqueous solution. Carbohydr Polym 83(1):270-276

6. Thanyacharoen T et al (2018) Development of a gallic acidloaded chitosan and polyvinyl alcohol hydrogel composite: release characteristics and antioxidant activity. Int J Biol Macromol 107:363-370

7. Thanyacharoen T et al (2017) The chemical composition and antioxidant and release properties of a black rice (Oryza sativa L.)-loaded chitosan and polyvinyl alcohol composite. J Mol Liq 248:1065-1070

8. Nguyen NT-P et al (2019) Stabilization of silver nanoparticles in chitosan and gelatin hydrogel and its applications. Mater Lett 248:241-245
9. George D, Maheswari PU, Begum KMMS (2019) Synergic formulation of onion peel quercetin loaded chitosan-cellulose hydrogel with green zinc oxide nanoparticles towards controlled release, biocompatibility, antimicrobial and anticancer activity. Int J Biol Macromol 132:784-794

10. Ahn J et al (2019) Network structure and enzymatic degradation of chitosan hydrogels determined by crosslinking methods. Carbohydr Polym 217:160-167

11. Ghavimi SAA et al (2019) Inductive co-crosslinking of cellulose nanocrystal/chitosan hydrogels for the treatment of vertebral compression fractures. Int J Biol Macromol 130:88-98

12. Bazrafshan Z, Stylios GK (2019) Spinnability of collagen as a biomimetic material: a review. Int J Biol Macromol 129:693-705

13. Sionkowska A et al (2016) 3D composites based on the blends of chitosan and collagen with the addition of hyaluronic acid. Int J Biol Macromol 89:442-448

14. Wang $M$ et al (2018) Preparation and characterization of novel poly (vinyl alcohol)/collagen double-network hydrogels. Int J Biol Macromol 118:41-48

15. Kudo $S$ et al (2017) Adsorption of water to collagen as studied using infrared (IR) microspectroscopy combined with relative humidity control system and quartz crystal microbalance. Appl Spectrosc 7:1621-1632

16. Beppu MM et al (2007) Crosslinking of chitosan membranes using glutaraldehyde: effect on ion permeability and water absorption. J Membr Sci 301(1):126-130

17. Fiejdasz $\mathrm{S}$ et al (2013) Biopolymer-based hydrogels as injectable materials for tissue repair scaffolds. Biomed Mater 8(3):035013

18. Gilarska A et al (2018) Collagen/chitosan/hyaluronic acid-based injectable hydrogels for tissue engineering applicationsdesign, physicochemical and biological characterization. Colloids Surf B 170:152-162

19. Lewandowska $\mathrm{K}$ et al (2016) Surface and thermal properties of collagen/hyaluronic acid blends containing chitosan. Int J Biol Macromol 92:371-376

20. Kaczmarek B, Sionkowska A, Stojkovska J (2018) Characterization of scaffolds based on chitosan and collagen with glycosaminoglycans and sodium alginate addition. Polym Test 68:229-232

21. Chiou SH, Wu WT (2004) Immobilization of Candida rugosa lipase on chitosan with activation of the hydroxyl groups. Biomaterials 25(2):197-204

22. Kaczmarek B, Sionkowska A, Skopinska-Wisniewska J (2018) Influence of glycosaminoglycans on the properties of thin films based on chitosan/collagen blends. J Mech Behav Biomed Mater 80:189-193

23. Freier T et al (2005) Controlling cell adhesion and degradation of chitosan films by N-acetylation. Biomaterials 26(29):5872-5878

24. Huang $Y$ et al (2005) In vitro characterization of chitosan-gelatin scaffolds for tissue engineering. Biomaterials 26(36):7616-7627

25. Liu Y, Ma L, Gao C (2012) Facile fabrication of the glutaraldehyde cross-linked collagen/chitosan porous scaffold for skin tissue engineering. Mater Sci Eng C 32(8):2361-2366

26. Padsalgikar AD (2017) Biological properties of plastics. In: Padsalgikar A (ed) Plastics in medical devices for cardiovascular applications. William Andrew, Burlington, pp 83-102

Publisher's Note Springer Nature remains neutral with regard to jurisdictional claims in published maps and institutional affiliations. 